\title{
Strong Coupling Constant from the Photon Structure Function
}

\author{
Simon Albino and Michael Klasen* \\ II. Institut für Theoretische Physik, Universität Hamburg, Luruper Chaussee 149, D-22761 Hamburg, Germany
}

\author{
Stefan Söldner-Rembold \\ FNAL, P.O. Box 500, MS 357, Batavia, Illinois 60510 \\ (Received 8 May 2002; published 29 August 2002)
}

\begin{abstract}
We extract the value of the strong coupling constant $\alpha_{s}$ from a single-parameter pointlike fit to the photon structure function $F_{2}^{\gamma}$ at large $x$ and $Q^{2}$ and from a first five-parameter full (pointlike and hadronic) fit to the complete $F_{2}^{\gamma}$ data set taken at PETRA, TRISTAN, and LEP. In next-to-leading order and the $\overline{\mathrm{MS}}$ renormalization and factorization schemes, we obtain $\alpha_{s}\left(m_{Z}\right)=0.1183 \pm$ $0.0050(\text { expt })_{-0.0028}^{+0.0029}$ (theor) (pointlike) and $\alpha_{s}\left(m_{Z}\right)=0.1198 \pm 0.0028(\text { expt })_{-0.0046}^{+0.0034}$ (theor) (pointlike and hadronic). We demonstrate that the data taken at LEP have reduced the experimental error by about a factor of 2, so that a competitive determination of $\alpha_{s}$ from $F_{2}^{\gamma}$ is now possible.
\end{abstract}

DOI: $10.1103 /$ PhysRevLett.89.122004

The theory of strong interactions, quantum chromodynamics (QCD), is one of the corner stones of the standard model of elementary particle physics. The precise determination of its fundamental parameter, the strong coupling constant $\alpha_{s}$, bears important implications for the validity not only of QCD itself, but also of even more fundamental theories, since these have to contain the standard model as an effective field theory in the lowenergy limit. A more fundamental theory, which might explain the size of the strong coupling constant, has yet to be established. Therefore, $\alpha_{s}$ must currently be extracted from experiment. Among the large variety of processes that have been used to this end, the most precise values have been obtained in $Z$ boson and $\tau$ decays at LEP, scaling violations in structure functions at HERA, and quarkonium decay branching fractions and lattice calculations of quarkonium mass splittings, leading — together with other, less precise measurements - to a current world average of $\alpha_{s}\left(m_{Z}\right)=0.1172 \pm 0.0020$ at the mass of the $Z$ boson, $m_{Z}=91.1876 \mathrm{GeV}$ [1].

When the photon structure function $F_{2}^{\gamma}$ was first discussed in the context of QCD, a precise determination of $\alpha_{s}$ quickly emerged as one of its most interesting applications. Because of the pointlike coupling of the photon to quarks, the leading order [LO, $\left.\mathcal{O}\left(\alpha / \alpha_{s}\right)\right]$ [2] and nextto-leading order [NLO, $\mathcal{O}(\alpha)$ ] [3] contributions to $F_{2}^{\gamma}\left(x, Q^{2}\right)$ are calculable in QCD perturbation theory, if the virtuality $Q$ in the deep-inelastic electron-photon scattering process is significantly larger than the asymptotic scale parameter $\Lambda$. Unfortunately, this pointlike contribution exhibits a power singularity at small Bjorken $x$ [4], which becomes rapidly stronger in higher orders [5]. The singularity can be regularized with a nonperturbative [6] or transverse-momentum [7] cutoff, but then the sensitivity to $\alpha_{s}$ is reduced and a dependence on the unphysical cutoff is introduced [8]. It is then necessary to fit both $\alpha_{s}$ and the cutoff to experimental data. Alternatively, the singularity can be canceled order
PACS numbers: 12.38.Bx, 12.38.Qk, 13.65.+i

by order in perturbation theory by retaining a hadronic boundary condition at a low starting scale $Q_{0}$ [9]. In this case it is necessary to fit $\alpha_{s}$ and the hadronic input to experimental data. However, the evolution of the hadronic input to the physical scale $Q$ is still predicted by perturbative QCD through inhomogeneous evolution equations [10], and the negligibility of the hadronic input can be tested a posteriori. Both methods have been applied in the past to PEP and PETRA data yielding $\Lambda_{\overline{\mathrm{MS}}}^{(4)}=$ $180_{-90}^{+100} \mathrm{MeV}$ [11] or $\alpha_{s}\left(m_{Z}\right)=0.108_{-0.010}^{+0.008}$. This value contributed to the world average in the 1988 [12], 1990 [13], and 1992 [14] issues of the Review of Particle Properties, but was then abandoned on the grounds that there were "no new results and the data do not contribute significantly to the average" [15]. Since then it has been believed [16-20] that the sensitivity of $F_{2}^{\gamma}$ to $\alpha_{s}$ is small.

In this Letter, we point out that over the last decade a wealth of new $F_{2}^{\gamma}$ data has been collected at the $e^{+} e^{-}$ colliders TRISTAN and LEP, which extends to high average values of $Q^{2},\left\langle Q^{2}\right\rangle \leq 780 \mathrm{GeV}^{2}$. We demonstrate that the new data improve the sensitivity of $F_{2}^{\gamma}$ to $\alpha_{s}$ significantly and that a single-parameter pointlike fit as well as a five-parameter full (pointlike and hadronic) fit to PETRA, TRISTAN, and LEP data yields results, which are not only consistent with the world average, but also have competitive experimental and theoretical errors.

We work in a fixed flavor number scheme with three active quark flavors $(u, d, s)$. It is well known [21] that for current measurements of $F_{2}^{\gamma}$ the available hadronic energy squared $W^{2}=Q^{2}(1-x) / x$ is not much larger than the production threshold $4 m_{h}^{2}$ of the heavy quarks $(h=$ $c, b, t)$, so that mass effects cannot be neglected and the massive, fixed order $\mathcal{O}(\alpha)$ expression for the BetheHeitler process $\gamma^{*}\left(Q^{2}\right) \gamma \rightarrow h \bar{h}$ [22] should be used instead of the massless, factorized $\mathcal{O}\left(\alpha / \alpha_{s}\right)$ expression. For a consistent NLO $[\mathcal{O}(\alpha)]$ analysis, we do not include the known [23], but numerically small, $\mathcal{O}\left(\alpha \alpha_{s}\right)$ corrections to the Bethe-Heitler process and omit the $\mathcal{O}\left(\alpha \alpha_{s}^{2}\right)$ 
contributions from the process $\gamma^{*}\left(Q^{2}\right) g \rightarrow h \bar{h}$. The heavy quark masses are not well constrained from measurements of $F_{2}^{\gamma}$. We adopt a charm quark mass of $m_{c}=1.5 \pm$ $0.1 \mathrm{GeV}$ in good agreement with recent precise determinations from threshold production at $e^{+} e^{-}$colliders [24]. We work in Mellin moment space, where the convolutions reduce to simple products, the evolution can be done analytically and without any approximations, and spurious higher order terms can be omitted. The resulting prediction for $F_{2}^{\gamma}$ is then converted back to $x$ space using a fast inverse Mellin transform with logarithmic mapping and fitted to experimental measurements with the multidimensional minimization algorithm MINUIT [25]. The quality of the fit is measured in terms of the $\chi^{2}$ value per degree of freedom, $\chi^{2} / \mathrm{DF}$, for all selected data points.

We include in our analysis all published measurements of $F_{2}^{\gamma}$ collected at the high-energy $e^{+} e^{-}$colliders PETRA [26-28], TRISTAN [29-31], and LEP [32-38]. If more than one set of statistically overlapping data exists, the most recent publication is used. We exclude from our fit the data published by the TPC $/ 2 \gamma$ Collaboration at PEP $[39,40]$, since several data points, mainly at low $x$, are inconsistent with measurements published by PLUTO [27], L3 [34], and OPAL [37] in the range $1.9<Q^{2}<$ $5.1 \mathrm{GeV}^{2}$. Data where the charm component has been subtracted are also discarded. Statistical uncertainties and correlations between data points due to the experimental unfolding are taken into account as provided by the experiments, while systematic uncertainties are assumed to be uncorrelated. Because of this assumption the values of $\chi^{2} / D F$ are expected to be on average slightly less than unity. If asymmetric errors are given by the experiments, the data points are taken at the center of the full error interval. Most experiments have not corrected for the finite virtuality of the target photon $P^{2}$. We neglect $P^{2}$ in this analysis, since usually $P^{2} \ll Q^{2}$.

For our pointlike fit, we identify the starting scale $Q_{0}$ with the asymptotic scale parameter $\Lambda$, so that the hadronic input vanishes automatically and only a singleparameter [ $\Lambda$, or equivalently $\left.\alpha_{s}\left(m_{Z}\right)\right]$ has to be fitted. As discussed above, this is justified only at large $x$ and $Q^{2}$, where the residue of the pointlike singularity is expected to be small. Therefore, we perform our single-parameter pointlike fit only to a subset of data points with $x \geq 0.45$ and $Q^{2} \geq 59 \mathrm{GeV}^{2}$. Very similar results are obtained with the widely used values of $Q_{0}=0.5, \ldots, 0.6 \mathrm{GeV}$ [16-18,20], while choosing $Q_{0}=1 \mathrm{GeV}$ significantly increases the value of $\chi^{2} / \mathrm{DF}$; two-parameter pointlike fits of $\alpha_{s}$ and $Q_{0}$ are driven to $Q_{0} \simeq \Lambda$. In the first three lines of Table I we list the $\chi^{2} / \mathrm{DF}$ and $\alpha_{s}\left(m_{Z}\right)$ values obtained in LO and NLO. The NLO fit is performed in two factorization schemes ( $\overline{\mathrm{MS}}$ and $\mathrm{DIS}_{\gamma}$ [21]) with different treatment of the pointlike Wilson coefficient in $F_{2}^{\gamma}$, but the numerical variation is found to be small. The total values of $\chi^{2} / \mathrm{DF}$ as well as those for the individual data sets (not shown) lie around unity or below, indicating that the pointlike photon structure function and the fitted values of $\alpha_{s}\left(m_{Z}\right)$ describe the data sets well within their statistical and systematic uncertainties. The experimental errors are determined by varying $\alpha_{s}\left(m_{Z}\right)$ until the total value of $\chi^{2}$ is increased by 1 unit. To estimate the theoretical error, we vary the charm quark mass as indicated above and follow the common convention of varying the factorization and renormalization scales by factors of 2 about their central value, the physical scale $Q$. We then add these three individual errors in quadrature. The LO value of $\alpha_{s}\left(m_{Z}\right)$ is consistent with the NLO value within the expected accuracy, $\mathcal{O}\left(\alpha_{s}^{2}\right)$, and the theoretical error is reduced from LO to NLO as expected. In the fourth line of Table I, we list the result of a fit without the LEP data. The experimental error is more than doubled, showing that the LEP data have considerably increased the sensitivity of $F_{2}^{\gamma}$ to $\alpha_{s}$ at high $x$ and $Q^{2}$. When data at all values of $x$, but very high $Q^{2}\left(Q^{2} \geq 284 \mathrm{GeV}^{2}\right)$ are fitted, the central value of $\alpha_{s}\left(m_{Z}\right)$ remains virtually unchanged (last line of Table I). At very high $Q^{2}$, the theoretical error drops by a factor of 2 , whereas the experimental error increases. Measurements of $F_{2}^{\gamma}$ at a future linear $e^{+} e^{-}$or $e \gamma$ collider like TESLA at very high values of $Q^{2}$ and with small experimental errors will therefore lead to even more precise determinations of $\alpha_{s}$.

The goodness of our pointlike fit may also be judged from Fig. 1, where the fitted data points are shown as full circles, while those that have been omitted from the fit are shown as open circles, and where the statistical and systematic errors have been added in quadrature. The theoretical curves are perturbatively stable, i.e., LO and NLO fits differ only by small amounts. The choice of the factorization scheme affects the region of very large $x$, but it has only a minor effect on the description of the data. Also shown in Fig. 1 is the hadronic contribution from a five-parameter NLO fit of the full photon structure function in the DIS $\gamma$ scheme. It clearly falls from small to large $x$ and $Q^{2}$ and amounts to only a few percent in the region that has been used in the pointlike fit.

For our full (pointlike and hadronic) fit, we start from the observations that $F_{2}^{\gamma}$ is dominated by the $u$-quark density in the photon and is only sensitive to the combined density of $d$ and $s$ quarks, whose contribution is furthermore suppressed by the smaller $d$ - and $s$-quark

TABLE I. $\chi^{2} / \mathrm{DF}$ and $\alpha_{s}\left(m_{Z}\right)$ values obtained in LO and NLO in the $\overline{\mathrm{MS}}$ and DIS $_{\gamma}$ factorization schemes with a single-parameter fit of the pointlike photon structure function $F_{2}^{\gamma}$. Also shown are the results obtained without LEP data and with very high $Q^{2}$ data.

\begin{tabular}{ccc}
\hline \hline Scheme & $\chi^{2} / \mathrm{DF}$ & $\alpha_{s}\left(m_{Z}\right)$ \\
\hline LO & $7.9 / 19$ & $0.1260 \pm 0.0055(\text { expt })_{-0.0055}^{+0.0061}$ (theor) \\
$\overline{\text { MS }}$ & $9.1 / 19$ & $0.1183 \pm 0.0050(\text { expt })_{-0.0028}^{+0.0029}$ (theor) \\
DIS $_{\gamma}$ & $8.1 / 19$ & $0.1195 \pm 0.0051(\text { expt })_{-0.0028}^{+0.0031}$ (theor) \\
w/o LEP & $3.2 / 7$ & $0.1244 \pm 0.0126(\text { expt })_{-0.0032}^{+0.0033}$ (theor) \\
High $Q^{2}$ & $11.9 / 8$ & $0.1159 \pm 0.0125(\text { expt })_{-0.0018}^{+0.0018}$ (theor) \\
\hline \hline
\end{tabular}




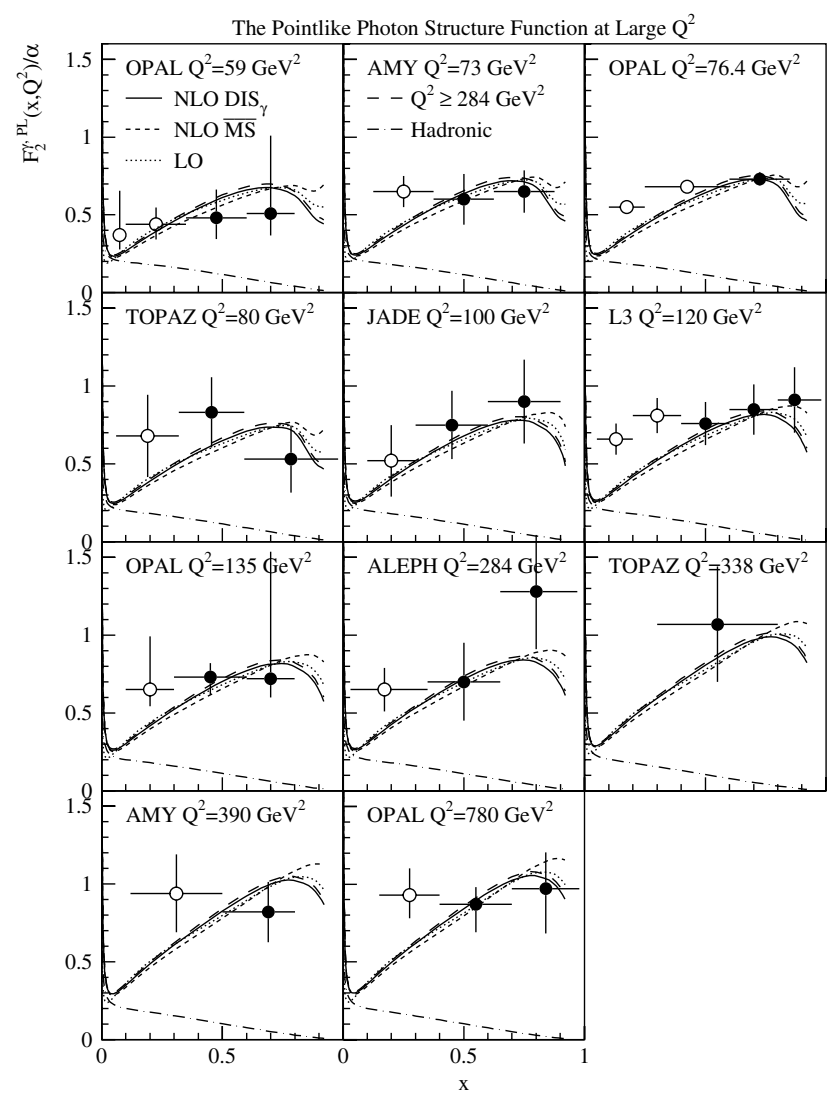

FIG. 1. Single-parameter fits of the pointlike photon structure function, compared to PETRA [26], TRISTAN [29,31], and LEP [32,34-36,38] data at large $Q^{2}$. The data points marked by open circles have not been used in the fits. Also shown is the hadronic contribution from a five-parameter NLO fit of the full photon structure function in the DIS ${ }_{\gamma}$ scheme.

charges. The gluon contributes to $F_{2}^{\gamma}$ in LO only through a rather weak coupling to the quark singlet density in the evolution equations. A consecutive fit of the $u$ quark, $d$ and $s$ quark, and gluon densities shows that only the first is well constrained by $F_{2}^{\gamma}$ data and that the fit does not improve, when more degrees of freedom are added. Therefore we do not impose a hadronic boundary condition for the gluon and assume that the hadronic fluctuations of the photon are insensitive to the quark charge, i.e., we identify the hadronic boundary conditions for $u$ quarks and $d$ and $s$ quarks at the starting scale $Q_{0}$. Together with $\alpha_{s}\left(m_{Z}\right)$ and $Q_{0}$, we then fit the parameters
$N, \alpha$, and $\beta$ of our ansatz $f_{u, d+s}^{\gamma}\left(x, Q_{0}^{2}\right)=N x^{\alpha}(1-x)^{\beta}$ to the full data set described above. In the first three lines of Table II we list the $Q_{0}, \chi^{2} / \mathrm{DF}$, and $\alpha_{s}\left(m_{Z}\right)$ values obtained with this five-parameter fit in LO and NLO. The starting scale $Q_{0}$ is perturbatively stable and is found to be close to the masses of the light vector mesons $\rho, \omega$, and $\phi$ in contrast to earlier claims that the perturbative evolution of $F_{2}^{\gamma}$ sets in only at rather high values of $Q_{0} \sim$ $2 \mathrm{GeV}$ [19]. The individual and total values of $\chi^{2} / \mathrm{DF}$ lie again around unity or below, so that the fitted full photon structure functions describe the full data set well within the experimental uncertainties. Note that the $\chi^{2}$ value for the four TPC $/ 2 \gamma$ points at $Q^{2}=2.8 \mathrm{GeV}^{2}$, which have not been used in the fits, is 18.0 and thus very large. The gluon density, generated with $f_{g}^{\gamma}\left(x, Q_{0}^{2}\right)=0$, turns out to be in good agreement with recent $\mathrm{H} 1$ dijet data [41]. The experimental errors on the values of $Q_{0}$ and $\alpha_{s}\left(m_{Z}\right)$ reflect an increase in $\chi^{2}$ by 1 unit, when all other fit parameters are kept fixed. Because of the larger number of data points in the full fit, the experimental error turns out much smaller than in the pointlike fit. When the full fit is performed without the LEP data (last line of Table II), the experimental error is almost doubled, i.e., the impact of the LEP data is again impressive. A fit to LEP data leads only to almost identical results as the full fit. The theoretical error in LO and without the LEP data gets a large asymmetric contribution from doubling the factorization scale, which is correlated with an increase in the value of $Q_{0}$ and which is reduced in the full NLO fit. Similar results as those listed in Table II are obtained, when only $u$ quarks are assigned a boundary condition.

In Fig. 2 we compare our results to the fitted $F_{2}^{\gamma}$ data in the region of low $x$ and $Q^{2}$. This region is clearly dominated by the hadronic contribution and by the impact of the LEP data. A fit without the LEP data results in a rise of $F_{2}^{\gamma}$ at low $x$, which is much too steep. The fits are perturbatively stable and the data are described almost equally well in the $\overline{\mathrm{MS}}$ and DIS ${ }_{\gamma}$ schemes.

Since the total error on $\alpha_{s}\left(m_{Z}\right)$ is smaller in the full fit than in the pointlike fit due to the larger number of data points, we adopt as our final result

$$
\alpha_{s}\left(m_{Z}\right)=0.1198 \pm 0.0054
$$

in NLO and the $\overline{\mathrm{MS}}$ scheme, where the larger theoretical error has been added to the experimental error in quadrature. While our total error is slightly larger than those

TABLE II. $Q_{0}, \chi^{2} / \mathrm{DF}$, and $\alpha_{s}\left(m_{Z}\right)$ values obtained in LO and NLO in the $\overline{\mathrm{MS}}$ and DIS $\gamma$ factorization schemes with a five-parameter fit of the hadronic photon structure function $F_{2}^{\gamma}$. Also shown are the results obtained without LEP data.

\begin{tabular}{cccc}
\hline \hline Scheme & $Q_{0} / \mathrm{GeV}$ & $\chi^{2} / \mathrm{DF}$ & $\alpha_{s}\left(m_{Z}\right)$ \\
\hline LO & $0.79 \pm 0.18$ & $121 / 129$ & $0.1475 \pm 0.0074(\text { expt })_{-0.0072}^{+0.014}$ (theor) \\
MS & $0.83 \pm 0.09$ & $118 / 129$ & $0.1198 \pm 0.0028(\text { expt })_{-0.0046}^{+0.0034}$ (theor) \\
DIS $_{\gamma}$ & $0.85 \pm 0.09$ & $115 / 129$ & $0.1216 \pm 0.0028(\text { expt })_{-0.0050}^{+0.0033}$ (theor) \\
w/o LEP & $0.46 \pm 0.10$ & $37 / 38$ & $0.1147 \pm 0.0047(\text { expt })_{-0.0033}^{+0.0282}$ (theor) \\
\hline \hline
\end{tabular}




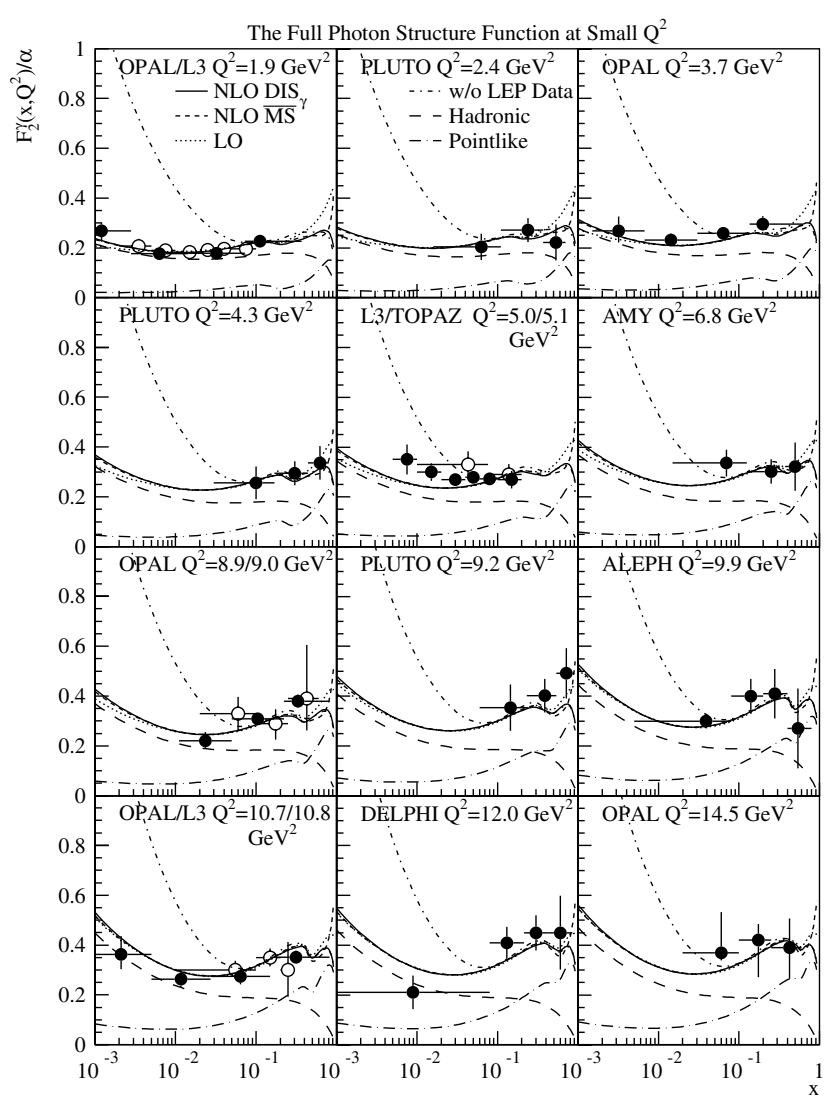

FIG. 2. Five-parameter fits of the full photon structure function, compared to data from PETRA [27], TRISTAN [30,31], and LEP [32-35,37] at small $Q^{2}$. The data points marked by open circles refer to the second experiment and/or $Q^{2}$ value. Also shown are the hadronic and pointlike contributions to the NLO fit in the DIS $_{\gamma}$ scheme.

obtained in $Z$ boson and $\tau$ decays at LEP, it is comparable to the errors obtained in deep-inelastic scattering at HERA and heavy quarkonium decays. This encourages us to combine our result with the current world average of $0.1172 \pm 0.0014[1]$ to a new world average

$$
\alpha_{s}\left(m_{Z}\right)=0.1175 \pm 0.0014
$$

where the errors are assumed to be uncorrelated.

In conclusion, we have for the first time fitted the now final PETRA, TRISTAN, and LEP data on the photon structure function $F_{2}^{\gamma}$ in NLO of perturbative QCD. We have extracted the value of the strong coupling constant $\alpha_{s}\left(m_{Z}\right)$ with competitive experimental and theoretical errors from a single-parameter pointlike fit to data at large $x$ and $Q^{2}$ and from a five-parameter full (pointlike and hadronic) fit at all $x$ and $Q^{2}$. Our analysis proves that the available $F_{2}^{\gamma}$ data contribute significantly to a precise determination of $\alpha_{s}$ and that future measurements of $F_{2}^{\gamma}$ at linear colliders will have a large impact.

We thank G. Kramer for many valuable discussions and a careful reading of the manuscript. S. A. and M. K. are supported by the Deutsche Forschungsgemeinschaft through Grant No. KL 1266/1-2.
*Electronic address: michael.klasen@desy.de

[1] K. Hagiwara et al., Phys. Rev. D 66, 010001 (2002).

[2] E. Witten, Nucl. Phys. B 120, 189 (1977).

[3] W. Bardeen and A. Buras, Phys. Rev. D 20, 166 (1979); 21, 2041(E) (1979).

[4] D. Duke and J. Owens, Phys. Rev. D 22, 2280 (1980).

[5] G. Rossi, Phys. Lett. B 130, 105 (1983).

[6] I. Antoniadis and G. Grunberg, Nucl. Phys. B 213, 445 (1983).

[7] J. Field, F. Kapusta, and L. Poggioli, Phys. Lett. B 181, 362 (1986).

[8] W. Frazer, Phys. Lett. B 194, 287 (1987).

[9] M. Glück and E. Reya, Phys. Rev. D 28, 2749 (1983).

[10] R. DeWitt, L. Jones, J. Sullivan, D. Willen, and H. Wyld, Phys. Rev. D 19, 2046 (1979); 20, 1751(E) (1979).

[11] W. Wagner, UC Davis Report No. UCD-86-29, 1986.

[12] G. Yost et al., Phys. Lett. B 204, 1 (1988).

[13] J. Hernandez et al., Phys. Lett. B 239, 1 (1990); 253, 524(E) (1990).

[14] K. Hikasa et al., Phys. Rev. D 45, S1 (1992); 46, 5210(E) (1992).

[15] L. Montanet et al., Phys. Rev. D 50, 1173 (1994).

[16] M. Glück, E. Reya, and A. Vogt, Phys. Rev. D 46, 1973 (1992).

[17] P. Aurenche, M. Fontannaz, and J. P. Guillet, Z. Phys. C 64, 621 (1994).

[18] G. Schuler and T. Sjöstrand, Z. Phys. C 68, 607 (1995).

[19] L. Gordon and J. Storrow, Nucl. Phys. B 489, 405 (1997).

[20] M. Glück, E. Reya, and I. Schienbein, Phys. Rev. D 60, 054019 (1999); 62, 019902(E) (1999).

[21] M. Glück, E. Reya, and A. Vogt, Phys. Rev. D 45, 3986 (1992).

[22] V. Budnev, I. Ginzburg, G. Meledin, and V. Serbo, Phys. Rep. 15C, 181 (1974).

[23] E. Laenen, S. Riemersma, J. Smith, and W. van Neerven, Phys. Rev. D 49, 5753 (1994).

[24] J. Kühn and M. Steinhauser, Nucl. Phys. B 619, 588 (2001).

[25] F. James and M. Roos, Comput. Phys. Commun. 10, 343 (1975).

[26] W. Bartel et al., Z. Phys. C 24, 231 (1984).

[27] C. Berger et al., Phys. Lett. B 142, 111 (1984); Nucl. Phys. B 281, 365 (1987).

[28] M. Althoff et al., Z. Phys. C 31, 527 (1986).

[29] S. Sahu et al., Phys. Lett. B 346, 208 (1995).

[30] T. Kojima et al., Phys. Lett. B 400, 395 (1997).

[31] K. Muramatsu et al., Phys. Lett. B 332, 477 (1994).

[32] R. Barate et al., Phys. Lett. B 458, 152 (1999).

[33] P. Abreu et al., Z. Phys. C 69, 223 (1996).

[34] M. Acciarri et al., Phys. Lett. B 436, 403 (1998); 447, 147 (1999); 483, 373 (2000).

[35] K. Ackerstaff et al., Phys. Lett. B 411, 387 (1997).

[36] K. Ackerstaff et al., Z. Phys. C 74, 33 (1997).

[37] G. Abbiendi et al., Eur. Phys. J. C 18, 15 (2000).

[38] G. Abbiendi et al., Phys. Lett. B 533, 207 (2002).

[39] H. Aihara et al., Phys. Rev. Lett. 58, 97 (1987).

[40] H. Aihara et al., Z. Phys. C 34, 1 (1987).

[41] C. Adloff et al., Phys. Lett. B 483, 36 (2000). 